\title{
CORRELATIVE QUANTITATIVE NANOMECHANICAL MAPPING AND CONFOCAL IMAGING OF LIVING CELLS BY SCANNING ION- CONDUCTANCE MICROSCOPY
}

Vasilii Kolmogorov ${ }^{1}$, Nikita Savin ${ }^{2}$, Aleksei Iakovlev ${ }^{1}$, Alexander Vaneev ${ }^{3}$, Yuri Efremov ${ }^{4}$, Svetlana Lavrushkina $^{5}$, Helena Lopatukhina ${ }^{6}$, Alexander Erofeev ${ }^{6}$, Natalia Klyachko ${ }^{5}$, Igor Kireev ${ }^{7}$, Alexander Majouga $^{8}$, Christopher Edwards ${ }^{9}$, Pavel Novak ${ }^{10}$, Yuri Korchev ${ }^{2}$ and Peter Gorelkin ${ }^{6}$

${ }^{1}$ National University of Science and Technology «MISiS», Moscow, Russia, Moskva, Russia, ${ }^{2}$ National University of Science and Technology "MISIS", Moscow, Russia, ${ }^{3}$ National University of Science and Technology «MISiS», Russia, ${ }^{4}$ Sechenov University, Moscow, Russia, Russia, ${ }^{5}$ Lomonosov Moscow State University, Moscow, Russia, Russia, ${ }^{6}$ National University of Science and Technology «MISiS», Moscow, Russia, Russia, ${ }^{7}$ Lomonosov Moscow State University, Moscow, Russia, United States, ${ }^{8}$ D. Mendeleev University of Chemical Technology, Russia, ${ }^{9}$ Imperial College London, London, United Kingdom, United Kingdom, ${ }^{10}$ National University of Science and Technology «MISiS», Moscow, Russia, United States

Quantitative nanomechanical mapping (QNM) of single cell via Scanning Ion-conductance Microscopy (SICM) is a novel method of studying cell mechanical properties. SICM allows to topography mapping with lateral and vertical nanoscale resolution, based on working principles. Moreover, it is possible to perform stiffness mapping simultaneously, due to applying force to living cell surface, whose nature is intrinsic colloidal pressure between nanopipette tip and cell membrane (Clarke et al., 2013, 2016). Nanoscale diameter of nanopipette tip allows to obtain cell QNM with nanoscale resolution and to avoid cell damage due to of small values of applying force and non-contact scanning.

As it was reported previously (Kolmogorov et al., 2021), SICM successfully used for detection of Young's modulus alteration of PC-3 and HT-1080 cells before and after treatment with drugs like paclitaxel or cytochalasin-D, which are change actin filaments or microtubules state. But in this particular work, we have demonstrated possibility of correlative QNM and confocal imaging, allowing to study living cell morphology, Young's modulus dynamic and live confocal visualization of cytoskeleton elements for single cell analysis. Correlative imaging showed significant changes of cell topography as well as changes to the Young's modulus and structure of actin filaments in confocal images. In the case of cell topography we observed a decrease in the height of the periphery of the cell but not at the central part after actin filaments disruption, which is explained by a higher concentration of actin located within the periphery of the cell. Then, a decrease in Young's modulus is clearly observable during Cyto-D treatment, as expected. Moreover, disruption of actin filaments is clearly observable at confocal image in comparison with control cell where structured filaments are seen. Also, aggregation of actin is observable, as expected. 
Thus, SICM technique can be successfully used for studying mechanical properties of living cells are important for various biological functions such as division, growth, differentiation, motility and tissue homeostasis.

This research was funded by The Russian Science Foundation, grant number № 19-19-00626.

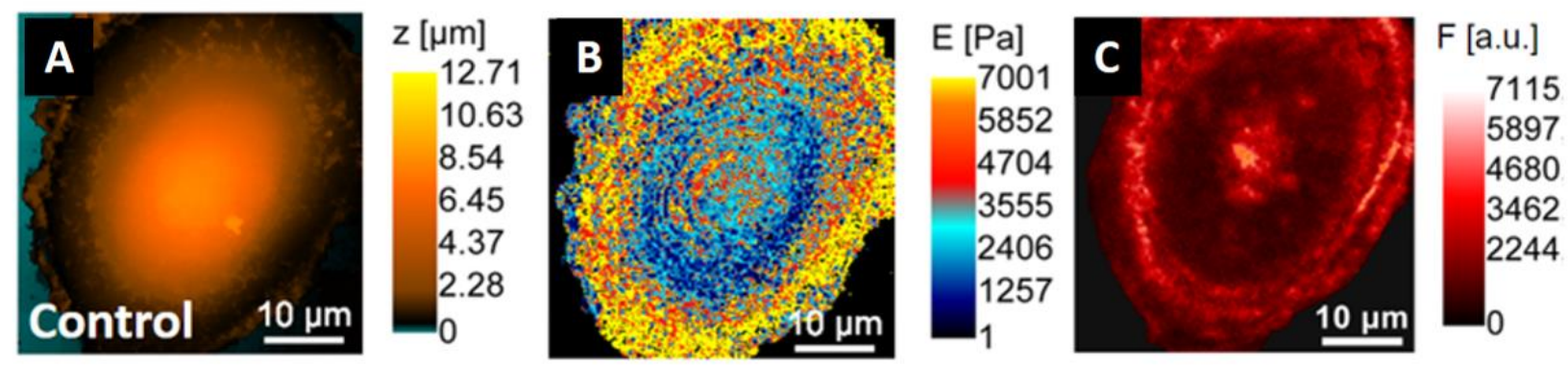

Figure 1. Fig 1 a, b and c - topography map, QNM and confocal image of actin filaments of living HT-1080 cell before Cyto-D treatment $(30 \mu \mathrm{M})$, respectively
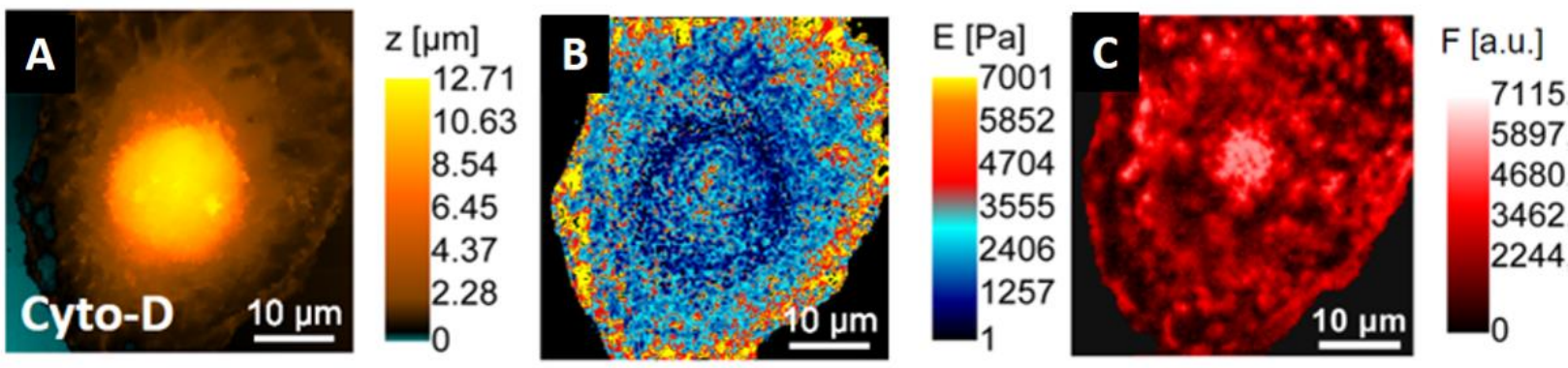

Figure 2. Fig 2 a, b and c - topography map, QNM and confocal image of actin filaments of living HT-1080 cell after Cyto-D treatment $(30 \mu \mathrm{M})$

\section{References}

Clarke, R. W., Novak, P., Zhukov, A., Tyler, E. J., Cano-Jaimez, M., Drews, A., Richards, O., Volynski, K., Bishop, C. \& Klenerman, D. (2016). Low Stress Ion Conductance Microscopy of Sub-Cellular Stiffness. Soft Matter 12, 7953-7958. www.rsc.org/softmatter (Accessed October 20, 2020).

Clarke, R. W., Zhukov, A., Richards, O., Johnson, N., Ostanin, V. \& Klenerman, D. (2013). Pipettesurface interaction: Current enhancement and intrinsic force. Journal of the American Chemical Society 135, 322-329. https://pubs.acs.org/doi/abs/10.1021/ja3094586 (Accessed October 21, 2020).

Kolmogorov, V., Vaneev, A., Efremov, Y., Savin, N., Iakovlev, A., Alova, A., Lavrushkina, S., Erofeev, A., Gorelkin, P., Klyachko, N., Kireev, I., Majouga, A., NovaK, P. \& Korchev, Y. (2021). Studying the Local Young's Modulus of PC-3 Cells Via Scanning Ion-Conductance Microscopy. Biophysical Journal 120, 162a. 